\title{
Respiratory supercomplexes: structure, function and assembly
}

\author{
Rasika Vartak, Christina Ann-Marie Porras, Yidong Bai ${ }^{\bowtie}$ \\ Department of Cellular and Structural Biology, University of Texas Health Science Center at San Antonio, San Antonio, TX 78229, \\ USA \\ $\triangle$ Correspondence: baiy@uthscsa.edu \\ Received April 21, 2013 Accepted May 23, 2013
}

\begin{abstract}
The mitochondrial respiratory chain consists of 5 enzyme complexes that are responsible for ATP generation. The paradigm of the electron transport chain as discrete enzymes diffused in the inner mitochondrial membrane has been replaced by the solid state supercomplex model wherein the respiratory complexes associate with each other to form supramolecular complexes. Defects in these supercomplexes, which have been shown to be functionally active and required for forming stable respiratory complexes, have been associated with many genetic and neurodegenerative disorders demonstrating their biomedical significance. In this review, we will summarize the functional and structural significance of supercomplexes and provide a comprehensive review of their assembly and the assembly factors currently known to play a role in this process.
\end{abstract}

\section{KEYWORDS supercomplex, mitochondrial, respiration}

\section{INTRODUCTION}

Mitochondria are ubiquitous organelles in eukaryotic cells whose primary function is to generate ATP through oxidative phosphorylation (Attardi and Schatz, 1988). The mitochondrial respiratory chain (MRC) is composed of five protein complexes: NADH-ubiquinone oxidoreductase as Complex I, succinate-ubiquinone oxidoreductase as Complex II, ubiquinonecytochrome-c oxidoreductase as Complex III, cytochromec oxidase as Complex IV, and ATP synthase as Complex V. Complex $\mathrm{I}$ is the entry point for electron transfer, accepting electrons from $\mathrm{NADH}_{2}$ which are transferred to Complex III via ubiquinone and then to Complex IV via cytochrome c. The proton transfer that accompanies the transport of electrons creates a gradient which provides the energy required to drive
ATP synthesis (Mitchell and Moyle, 1968). There have always been two viewpoints of how these respiratory enzymes exist in the inner mitochondrial membrane. While one envisions the respiratory enzymes as free floating in the inner mitochondrial membrane with cytochrome $\mathrm{c}$ and ubiquinone as connecting molecules; the other predicts that respiratory enzymes exist in a solid complex composed of varying ratios of different complexes associating with each other to form supercomplexes. While the earlier viewpoint had more support and evidence backing it in the past, the availability of sensitive biochemical assays is slowly replacing the textbook model of independent complexes floating in the inner mitochondrial membrane in favor of a MRC model composed of stable supercomplexes operating as the functional units of respiration. The importance of supercomplexes in the pathology of various human diseases is slowly becoming apparent, with reduced and destabilized supercomplexes being observed in various genetic, aging and neurodegenerative disorders.

\section{SUPERCOMPLEXES: UNITS OF RESPIRATION}

The idea that respiratory enzymes occur in a supramolecular complex with each other is hardly new; Chance and Williams (1955) were the first to propose that the respiratory enzymes existed as a single unit of respiration. Further research; however, gave rise to evidence that contrasted with the solid state model: when individual respiratory complexes were isolated they were found to possess biochemical activity (Hatefi et al., 1962), which was unexpected as the solid state model hypothesized that respiratory activity existed only if the enzymes were present as a complex. Additionally, the use of fluorescence recovery after photobleaching (FRAP) and flux control analysis, demonstrated that the electron transfer between different reducing equivalents mediated by cytochrome $\mathrm{c}$ and ubiquinone required multiple collisions and occurred across a long range distance of $36 \mathrm{~nm}$ (Hackenbrock et al., 1986; Chazotte and 
Hackenbrock, 1988; Gupte and Hackenbrock, 1988) indicating that the respiratory enzymes were not in close contact with each other as the solid state model predicted. The electron transfer thus did not seem to require the formation of respiratory enzyme complexes. Even though most of the evidence backed the 'liquid state' model, it could not however explain the isolation of Complex III/IV supercomplexes from bacteria (Berry and Trumpower, 1985; Sone et al., 1987; Iwasaki et al., 1995) and yeast (Bruel et al., 1996; Boumans et al., 1998). Finally, using blue native polyacrylamide gel electrophoresis (BNPAGE) and milder detergents to isolate mitochondrial membrane proteins, studies from bovine mitochondria showed Complex I, III and IV in supercomplexes, with almost all of Complex I observed in supercomplexes rather than individual units (Schägger and Pfeiffer, 2000). The most common supercomplexes documented are Complex I/III , Complex I/IIIn/IV and Complex III/IV $\mathrm{n}$ (Berry and Trumpower, 1985; Schägger and Pfeiffer, 2001; Stroh et al., 2004; Dudkina et al., 2011) (Fig. $1 \mathrm{~B}$ and $1 \mathrm{C}$ ). Most of Complex II was found in a free, non-associated form in plant as well as mammalian mitochondria, while only a small proportion associated with supercomplex I/III/IV (Eubel et al., 2003; Acín-Pérez et al., 2008; Muster et al., 2010) (Fig. 1A). While Complex $\mathrm{V}$ as dimer co-migrates with other supercomplexes but rarely as part of supercomplexes. The following two sections will detail the functional as well as structural significance of supercomplexes while the rest of the review will attempt to delineate what is currently understood about supercomplex assembly and the assembly factors involved.

\section{FUNCTIONAL SIGNIFICANCE OF SUPERCOMPLEXES}

The consistent and repeated observations of respiratory complexes associating with each other to form supercomplexes has slowly replaced the idea of a 'fluid state' model of the electron transport chain. However, while plenty of structural evidence was available for the existence of supercomplexes, mainly in the form of electrophoretic patterns on Blue Native PAGE or density gradient centrifugations, not enough evidence was available to indicate their functional significance. Early evidence came from Hatefi et al. who successfully isolated intact Complex I-III particles and established that maximal enzyme activity can be accomplished by reconstituting a mixture of Complex I and Complex III. These reconstituted complexes were stable, and their activities were very similar to particles directly isolated from mitochondria (Hatefi, 1961, 1978). The theory of electron transfer being multi-collisional and long range was countered by evidence in yeast which demonstrated that 'pool behavior' of cytochrome $c$ and ubiquinone did not exist under physiological conditions and was introduced by the addition of chaotropic reagents that dissociated the individual enzymes from the single unit respiratory complex (Boumans et al., 1998). Using flux control kinetics, it was shown that Complex I and Complex III both exert rate control on NADH oxidation implying that they are present in a single complex. The control exerted by Complex IV however over NADH oxidation was relatively weak indicating that Complex IV remained in a free form state. This study also sought to reconcile previous counteracting studies of the existence of cytochrome $c$ and ubiquinone pools, predicting that these molecules may remain in free form or associate with supercomplexes depending on the metabolic needs of the mitochondria (Bianchi et al., 2004). Last but not least, respiratory active supercomplexes containing Complex I/II/III/IV and I/III/IV were isolated and shown to respire in a Clarke type oxygen electrode (Acín-Pérez et al., 2008). Termed respirasomes owing to their ability to form functional units of respiration, they were found to contain both cytochrome $\mathrm{c}$ and $\mathrm{CoQ}$. Interestingly, cybrids made by fusing one cell type with Complex IV deficiency and another cell type with Complex III deficiency completely recovered respiration by complementation and this recovery of respiration correlated with the presence of Complex I/III/IV supercomplexes (D'Aurelio et al., 2006). While all of these reports point to a more efficient electron transport when respiratory enzymes are present as supercomplexes, a few recent studies still questioned their functional significance (Trouillard et al., 2011). The existence of supercomplexes such as III/IV as well as I/III also pose a challenge: what roles could these supercomplexes have if they are not simply artifacts of electrophoresis? It is possible that at any given point in time the respiratory complexes exist as either supercomplexes or as individual complexes depending on the metabolic needs of the cell or the amount of phospholipids in the inner mitochondrial membrane. In such a scenario, supercomplexes I/III and III/IV may act as intermediate supercomplexes which then associate together to form higher order supercomplexes (Acín-Pérez et al., 2008). As discussed in the following sequence, supercomplex I/III may be necessary to preserve the stability of Complex I. There are also reports of a functional I/III supercomplex. While electron transfer was not found to require the formation of I/III supercomplex, flux analysis demonstrated that I/III supercomplex did participate in electron transfer and substrate channeling (Bianchi et al., 2004) and provided a kinetic advantage with a greater rate of NADH cytochrome c reductase activity (Genova et al., 2008). It has also been suggested that formation of supercomplexes may reduce oxidative damage by sequestering vulnerable sites. Indeed, decreased supercomplexes have been observed in pathologies underlined by oxidative stress (Rosca et al., 2008; Gómez et al., 2009). Due to the vast variations observed in the amount and content of supercomplexes between different cell types, a 'plasticity model' was hypothesized (Acín-Pérez et al., 2008) wherein differences in the cell types and physiological states give rise to different combinations of respiratory supercomplexes (Fig. 1). Evidence for this has been observed in plant mitochondria wherein the composition of supercomplexes changed with oxygen availability (Ramírez-Aguilar et al., 2011). Thus, different metabolic needs for different organisms and tissue types may dictate the existence of supercomplexes at any given time allowing the entire respiratory chain the flexibility to accommodate the ATP demands of the cell. It would therefore 


\section{Complex I Complex II Complex III Complex IV}

A. Electron transport in Supercomplex I/II/III/IV

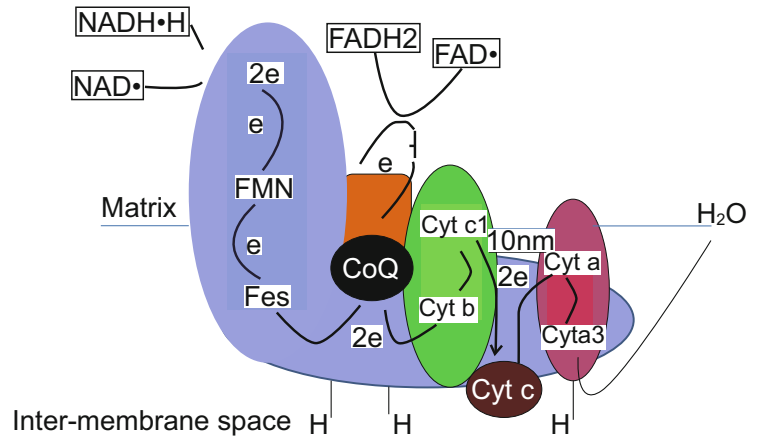

B. Electron transport in Supercomplex I/III/IV

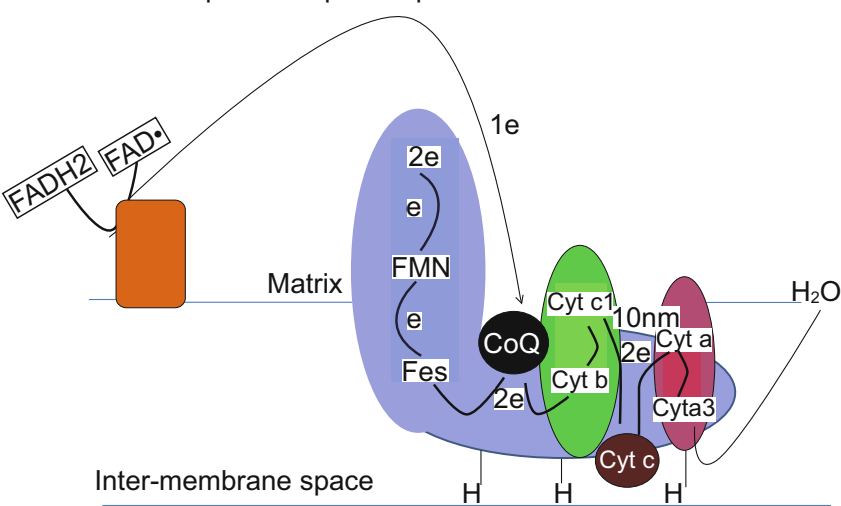

C. Electron transport in Supercomplex I/III

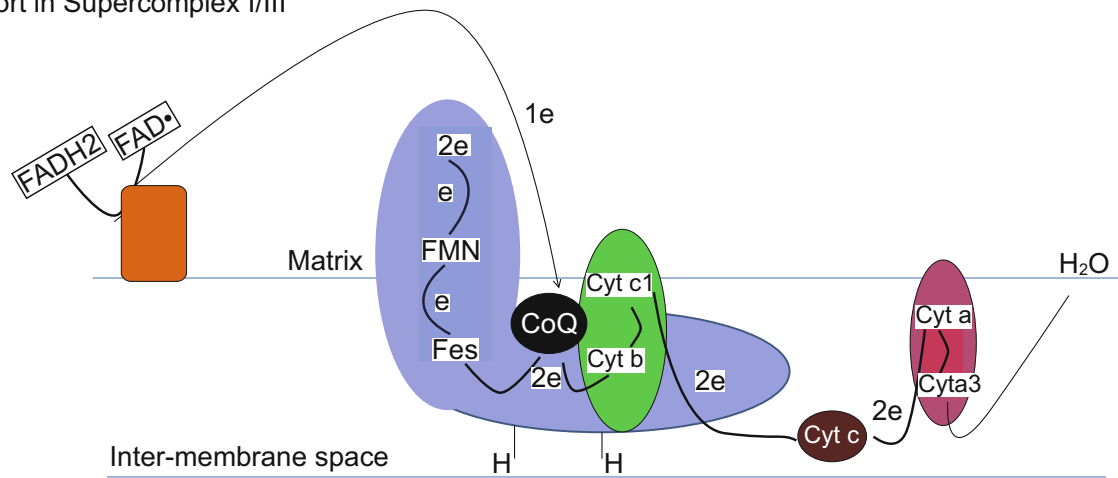

Figure 1. Electron transport through different forms of functional supercomplexes. (A) Electron transport through supercomplex I/ II/III/IV (respirasome) (B) Electron transport through supercomplex I/III and (C) Electron transport through supercomplex I/III/IV.

be of interest to study the composition of supercomplexes in various tissues and understand how the metabolic and stress landscape alters supercomplexes in these tissues.

\section{STRUCTURAL SIGNIFICANCE AND ASSEMBLY OF SUPERCOMPLEXES}

The structural implications of supercomplexes have been evident for some time. In two separate studies, Complex I assembly was found to depend on Complex III (Acin-Perez et al., 2004) as well as Complex IV (Diaz et al., 2006; Li et al., 2007). While Complex I was found to be unstable in the absence of Complex III, lack of Complex IV totally abrogated assembly of Complex I (Li et al., 2007). The lack of Complex I however was not found to affect either Complex III or Complex IV (Acin-Perez et al., 2004; Li et al., 2007). These are early evidences of the structural significance of supercomplexes which were reproduced in the bacterial model system, $P$. denitrificans wherein Complex I was found to be stabilized in a supercomplex with Complex III and Complex IV (Stroh et al., 2004). Surprisingly, in patient cells with mutations in Complex IV COX genes resulting in reduced levels of the subunits, most of Complex IV was preferentially incorporated into supercomplexes leaving less or no free Complex IV (Lazarou et al., 2009). Thus supercomplex formation not only seems to allow greater substrate channeling and electron transfer but also confers structural stability to the respiratory enzymes.

High resolution electron microscopy of different supercomplexes in plants as well as mammalian mitochondria has been of great use in trying to resolve the structures of various supercomplexes in order to understand this structural and functional dependence. High resolution of I/IIIn supercomplex in plant Arabidopsis thaliana showed for the first time that Complex III associates with Complex I by interacting with its membrane arm (Dudkina et al., 2005). The study also predicted that this association might help ubiquinone bind to Complex I, which occurs above the junction of the membrane and matrix arm, leading to a more efficient electron/proton flow and the reduction in reactive oxygen species production. Single particle electron microscopy using a 3D reconstructed map superimposed on the supercomplex of $Y$. lipolytica, during subsequent studies in bovine mitochondria, confirmed this interaction while also resolving the structure of Complex $\mathrm{I} / \mathrm{II}_{2} / \mathrm{IV}$ revealing that Complex IV is present adjacent to the Complex III dimer at the distal tip of 


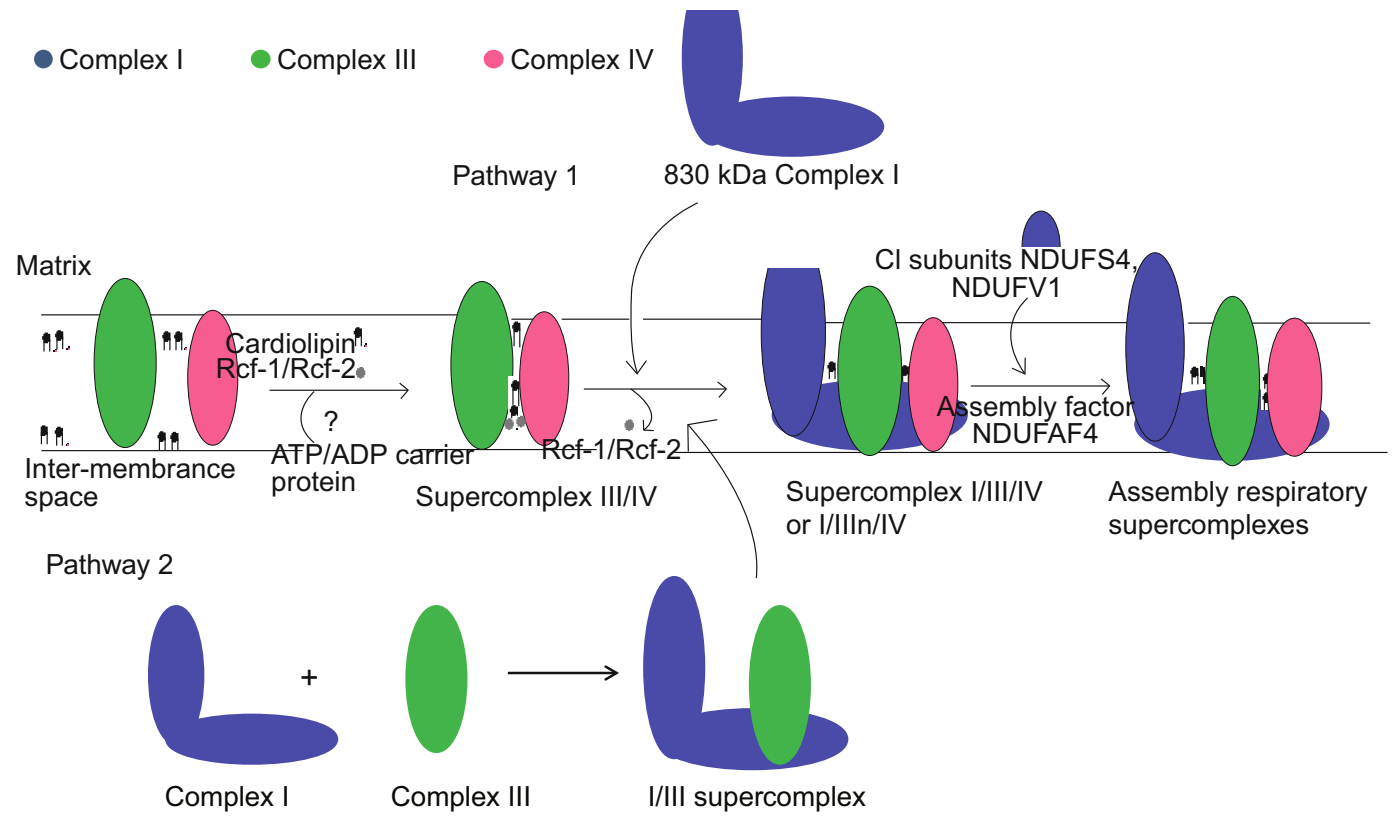

Figure 2. Assembly pathway for supercomplexes: Two proposed pathways for the assembly of respiratory supercomplexes. The first pathway (upper) details formation of I/III/IV supercomplex and the roles of cardiolipin and Rcf-1/2 in the assembly process. The second pathway (lower) details formation of the $\mathrm{I} / \mathrm{II} \mathrm{I}_{\mathrm{n}} / \mathrm{IV}$ supercomplex with $\mathrm{I} / \mathrm{III}$ supercomplex joining III/IV supercomplex forming supercomplexes with Complex III dimer.

the Complex I membrane arm (Schäfer et al., 2006; Althoff et al., 2011; Dudkina et al., 2011). Additionally, ubiquinone binding sites were found between Complex I and III at the interphase of the membrane and peripheral arm of Complex I while cytochrome $\mathrm{c}$ diffusion distance was found to be $10 \mathrm{~nm}$ or less (Dudkina et al., 2005; Heinemeyer et al., 2007). It was also observed that the complexes are in loose association with one another with the spaces presumably filled by membrane lipids such as cardiolipin to keep the complexes stable or to enhance the diffusion of ubiquinone and cytochrome c. Thus these findings lend credibility to the functional and structural significance of supercomplexes.

\section{SUPERCOMPLEX ASSEMBLY AND ASSEMBLY FACTORS}

This dependence of Complex I on other complexes led to the hypothesis that supercomplex assembly formation was necessary for the stability of assembled, individual respiratory Complex I which in turn had major biomedical implications (Budde et al., 2000; D’Aurelio et al., 2006; Morán et al., 2010). Understanding the mechanism of supercomplex assembly has therefore been the focal point of research in the past few years. Because Complex I stability was found to be dependent on the assembly of supercomplexes, it was hypothesized that supercomplex assembly follows the assembly of individual respiratory complexes. Labeling of mitochondrial translational products indicated that there is sequential incorporation of mtDNA encoded subunits into respective complexes followed by super- complex assembly. Thus there seemed to be a temporal gap in the assembly of individual complexes and supercomplexes (Acín-Pérez et al., 2008). However, a more extensive study in Neurospora Crassa showed that Complex I assembly and synthesis was very closely linked with supercomplex formation as the formation of Complex I/III supercomplex was observed to occur before Complex I was formed in its entirety (Marques et al., 2007) (Fig. 2). It was elegantly shown in mouse mitochondria that Complex I, encompassing the mtDNA encoded subunits, assembles independently to the $830 \mathrm{kDa}$, high molecular weight. However, association of Complex IV and Complex III subunits, in that order, with the assembled $830-\mathrm{kDa}$ Complex $\mathrm{I}$ is required for the incorporation of the remaining Complex I subunits such as NDUFS4 and NDUFV1. Thus assembly and not the just stability of Complex I may require an association with Complex III and IV indicating that supercomplex assembly precedes the assembly of individual respiratory complexes (Moreno-Lastres et al., 2012). Interestingly, this study also observed a lack of assembled Complex I as an individual respiratory enzyme; almost all of the Complex I was present in the supercomplex form indicating the significant role of the detergent concentration in isolating individual complexes as well as supercomplexes.

Assembly factors that aid in the assembly of various respiratory enzymes have been of immense significance not only in trying to understand complex assembly processes but also from a biomedical point of view. Many assembly factors for complexes I, III and IV were found due to mutations identified in patients that caused a deficiency of respiratory enzymes 
leading to mitochondrial disorders (Budde et al., 2000; Dunning et al., 2007; Fernandez-Vizarra et al., 2007; Saada et al., 2008; Sugiana et al., 2008; Ghezzi et al., 2011). The current hypothesis for supercomplex assembly is two-fold: either there are exclusive assembly factors that help assemble supercomplexes after assembly of individual complexes or the assembly factors between different respiratory enzymes are shared. There are merits and evidences for both lines of reasoning.

\section{Cardiolipin}

The earliest assembly factor thought to be involved in the supercomplex assembly was identified to cause a metabolic disorder. Barth syndrome is characterized by cardiomyopathy, skeletal myopathy and neutropenia (Barth et al., 1983). It is an X-linked disorder caused by mutations in the taffazin (TAZ) gene that lead to reduced production of a membrane lipid called cardiolipin (Bione et al., 1996; Orstavik et al., 1998; Vreken et al., 2000). Cardiolipin is primarily found in mitochondrial membranes (Fleischer et al., 1967) and a decrease in cardiolipin was found to be associated with decreased membrane potential, ATP synthesis and overall mitochondrial function (Santiago et al., 1973; Jiang et al., 2000; Gohil et al., 2004). The link between cardiolipin and supercomplex assembly became apparent with the finding that supercomplexes are destabilized in patients with Barth syndrome (McKenzie et al., 2006). It was subsequently determined biochemically as well as through electron microscopy that cardiolipin physically binds to Complex I, Complex III, Complex IV and Complex V in the inner mitochondrial membrane (Fry and Green, 1981; Arnarez et al., 2013a, 2013b), with as many as 6 binding sites identified in Complex III and IV. In yeast, cardiolipin was needed for the formation of Complex III/IV supercomplexes (Zhang et al., 2005; Bazán et al., 2013) as well as for the transfer of electrons from Complex I to Complex III (Fry and Green, 1981). Thus, it is possible that cardiolipin in the inner mitochondrial membrane conserves the stability of supercomplexes and maintains efficient electron transfer between different complexes.

\section{Rcf-1 and Rcf-2}

These are isoforms of Hypoxia induced genes (Hig-1) whose functions were unknown except that they were present in the mitochondrial inner membrane and their absence caused mitochondrial dysfunction (Wang et al., 2006; Hess et al., 2009). Recently, Rcf-1 and Rcf-2 were found to associate directly with Complex IV and indirectly with Complex III. Upon knockdown, Rcf-1 and Rcf-2 were found to affect the levels of supercomplex III/IV in yeast cells (Strogolova et al., 2012). The mammalian homolog of Rcf-1, Hig2A, was also found to associate with supercomplex III/IV and facilitate their assembly/stability. Hig2A knockdown was also found to reduce all supercomplexes containing Complex IV, including I/IIIn/IV and I/III/IV/V supercomplexes (Chen et al., 2012). Interestingly, in both of these studies, knockdown of these factors reduced only supercomplex levels and not levels of individual assembled complexes, making them true assembly factors for supercomplexes.

\section{ATPIADP carrier protein (AAC)}

AAC1 and AAC2 are multi-subunit proteins present in the inner mitochondrial membrane and are responsible for the transport of ATP across the mitochondrial membrane (Klingenberg, 1989; Gawaz et al., 1990). Deletion of these proteins in yeast cells gives rise to lethality suggesting that $A A C$ proteins have cellular functions other than ATP/ADP transport (Chen, 2004). Using $2 \mathrm{D}$ electrophoresis, it was shown that AAC2 not only interacts but also co-purifies with the Complex III/IV supercomplex and AAC2 null yeast cells showed decreased Complex $\mathrm{III}_{2} / \mathrm{IV}_{2}$ levels (Dienhart and Stuart, 2008). Whether the mammalian homologue participates in supercomplex assembly is however not investigated, and its implications in mammalian supercomplex assembly are unknown.

\section{Other putative supercomplex assembly factors}

A few other proteins and lipids have been hypothesized to participate in assembly and stability of supercomplexes. However, firm evidence for their role in supercomplex assembly/stability is currently lacking. Prohibitins (PHB) are a conserved family of proteins present in the inner mitochondrial membrane (Berger and Yaffe, 1998). PHB1 and PHB2 are the most commonly found prohibitins in the mitochondria. Past research has suggested that prohibitins are scaffold-like proteins (Merkwirth et al., 2012) that are involved in mitochondrial function, fission/fusion and mitochondrial biogenesis (Ahn et al., 2006; Schleicher et al., 2008). Prohibitin has been found to interact with Complex IV (Strub et al., 2011) and co-migrates with supercomplex $\mathrm{III}_{\mathrm{n}} / \mathrm{IV}_{\mathrm{n}}$ (Marques et al., 2007). There is a focus on phospholipids other than cardiolipin that can bind to and stabilize respiratory supercomplexes (Wenz et al., 2009). A study comparing roles of phosphatidylethanolamine (PE) and cardiolipin showed that both were important in the formation and activity of supercomplexes (Böttinger et al., 2012). While the study did not see a decrease in supercomplexes when PE was depleted, there was a change in the pattern of supercomplexes formed, suggesting a role for different phospholipids in supercomplex assembly and stability. Since it has been reported that assembly of supercomplexes precedes assembly of Complex I, another hypothesis is that assembly factors required for the last stage of Complex I assembly may in fact be considered supercomplex assembly factors since Complex III and Complex IV have already assembled with Complex I. Thus, NDUFAF2, a Complex I assembly factor that binds to the $830-K D a$ Complex I to incorporate the rest of the nuclear encoded subunits, might also be considered a supercomplex assembly factor (Ogilvie, 2005; Vogel et al., 2007).

\section{FUTURE DIRECTIONS}

Supercomplexes and their role in respiration and mitochondrial function have surfaced only recently. The role of supercomplex 
assembly defects in diseases such as Barth syndrome, aging, other Complex I deficiency disorders such as cardiac myopathies and neurodegenerative disorders is slowly coming to light, and it is of biomedical significance that the assembly and function of supercomplexes is understood clearly. Extensive studies are required to understand how altered supercomplexes translate into mitochondrial dysfunction and how this dysfunction can influence the development and progression of various pathologies. Supercomplex dysfunction may have at least two different impacts on cell survival. Because supercomplexes are believed to be the functional unit of respiration, it follows that defects in either their assembly or stability will decrease the efficiency of the electron transport chain subsequently decreasing oxidative phosphorylation and ATP production. Moreover, the 'solid state' model of the electron transport chain consisting of supercomplex is believed to reduce the generation of reactive oxygen species whose overproduction can have disastrous consequences for the cell (Dudkina et al., 2005; Rosca et al., 2008; Gómez et al., 2009). Therefore supercomplex deficiencies may result in a more liquid electron transport chain and ROS overproduction. Either of these consequences should theoretically impact tissues that are more reliant on oxidative phosphorylation than other tissues which preferentially utilize glycolysis as a means for ATP production. Thus, nervous and muscle tissues may be particularly vulnerable as exemplified by the cardiac and skeletal myopathies observed in Barth Syndrome and the connection between supercomplex deficiencies and both neurodegeneration disorders and aging (Barth et al., 1983; Gómez et al., 2009; Merkwirth et al., 2012). In particular there is a need to further characterize the supercomplex assembly factors that are known but also to identify novel assembly factors that may participate in disease pathology. Comprehensive loss of respiratory function due to mutations in subunits such as Cox IV (Complex IV) and RISP (Complex III) is now understood to arise due to the loss of supercomplexes, as these subunits associate with Complex I to make a stable supercomplex (Fernandez-Vizarra et al., 2007; Morán et al., 2010). Considerable attention has been given to understanding the structure and function of Complex III/IV supercomplexes. The same needs to be done for supercomplexes containing Complex I; especially since they are the true 'respirasomes' and will affect mitochondrial function to a large extent. While working with supercomplexes containing Complex I may be difficult due to their large sizes, innovative methods such as subtractive proteomics and phylogenetic profiling have been used to identify Complex I specific proteins which may be involved in Complex I function and assembly (Pagliarini et al., 2008). Similar methods can be applied to identify more proteins involved in supercomplex assembly and function. Due to the advent of new proteomics approaches, allowing better resolution of mass spectrometry, as well as new biochemical techniques such as the use of new and improved electrophoresis methods (large pore and clear native gel); the identification of new assembly factors has become more feasible. Taking advantage of this new technology is the next big step in un- derstanding how supercomplexes assemble and function and how they can cause mitochondrial dysfunction.

\section{ACKNOWLEDGEMENTS}

The relevant work carried out in the authors' laboratory has been supported by grants from National Institute of Health (R21 NS072777) and the Morrison Trust.

\section{ABBREVIATIONS}

AAC, ATPIADP carrier protein; BNPAGE, blue native polyacrylamide gel electrophoresis; FRAP, fluorescence recovery after photobleaching; $\mathrm{PHB}$, prohibitin

\section{COMPLIANCE WITH ETHICS GUIDELINES}

Authors Rasika Vartak, Christina Porras and Yidong Bai declare no conflict of interest.

\section{REFERENCES}

Acin-Perez, R., Bayona-Bafaluy, M.P., Fernandez-Silva, P., MorenoLoshuertos, R., Perez-Martos, A., Bruno, C., Moraes, C.T., and Enriquez, J.A. (2004). Respiratory complex III is required to maintain complex i in mammalian mitochondria. Mol Cell 13, 805-815.

Acín-Pérez, R., Fernández-Silva, P., Peleato, M.L., Pérez-Martos, A., and Enriquez, J.A. (2008). Respiratory active mitochondrial supercomplexes. Mol Cell 32, 529-539.

Ahn, C.S., Lee, J.H., Reum Hwang, A., Kim, W.T., and Pai, H.-S. (2006). Prohibitin is involved in mitochondrial biogenesis in plants. Plant $\mathrm{J}$ 46, 658-667.

Althoff, T., Mills, D.J., Popot, J.-L., and Kühlbrandt, W. (2011). Arrangement of electron transport chain components in bovine mitochondrial supercomplex I1III2IV1. EMBO J 30, 4652-4664.

Arnarez, C., Mazat, J.-P., Elezgaray, J., Marrink, S.-J., and Periole, X. (2013a). Evidence for cardiolipin binding sites on the membraneexposed surface of the cytochrome bc(1). J Am Chem Soc 135, 3112-3120.

Arnarez, C., Marrink, S.J., and Periole, X. (2013b). Identification of cardiolipin binding sites on cytochrome $c$ oxidase at the entrance of proton channels. Sci Rep 3, 1263.

Attardi, G., and Schatz, G. (1988). Biogenesis of mitochondria. Annu Rev Cell Biol 4, 289-333.

Barth, P.G., Scholte, H.R., Berden, J.A., Van der Klei-Van Moorsel, J.M., Luyt-Houwen, I.E., Van't Veer-Korthof, E.T., Van der Harten, J.J., and Sobotka-Plojhar, M.A. (1983). An X-linked mitochondrial disease affecting cardiac muscle, skeletal muscle and neutrophil leucocytes. J Neurol Sci 62, 327-355.

Bazán, S., Mileykovskaya, E., Mallampalli, V.K.P.S., Heacock, P., Sparagna, G.C., and Dowhan, W. (2013). Cardiolipin-dependent reconstitution of respiratory supercomplexes from purified saccharomyces cerevisiae complexes III and IV. J Biol Chem 288, 401-411.

Berger, K.H., and Yaffe, M.P. (1998). Prohibitin family members interact genetically with mitochondrial inheritance components in Saccharomyces cerevisiae. Mol Cell Biol 18, 4043-4052.

Berry, E.A., and Trumpower, B.L. (1985). Isolation of ubiquinol oxidase from Paracoccus denitrificans and resolution into cytochrome bc1 
and cytochrome c-aa3 complexes. J Biol Chem 260, 2458-2467.

Bianchi, C., Genova, M.L., Parenti Castelli, G., and Lenaz, G. (2004). The mitochondrial respiratory chain is partially organized in a supercomplex assembly: kinetic evidence using flux control analysis. J Biol Chem 279, 36562-36569.

Bione, S., D'Adamo, P., Maestrini, E., Gedeon, A.K., Bolhuis, P.A., and Toniolo, D. (1996). A novel X-linked gene, G4.5. is responsible for Barth syndrome. Nat Genet 12, 385-389.

Böttinger, L., Horvath, S.E., Kleinschroth, T., Hunte, C., Daum, G., Pfanner, N., and Becker, T. (2012). Phosphatidylethanolamine and cardiolipin differentially affect the stability of mitochondrial respiratory chain supercomplexes. J Mol Biol 423, 677-686.

Boumans, H., Grivell, L.A., and Berden, J.A. (1998). The respiratory chain in yeast behaves as a single functional unit. J Biol Chem 273, 4872-4877.

Bruel, C., Brasseur, R., and Trumpower, B.L. (1996). Subunit 8 of the Saccharomyces cerevisiae cytochrome bc1 Complex Interacts with succinate-ubiquinone reductase complex. J Bioenerg Biomembr 28, 59-68.

Budde, S.M., Van den Heuvel, L.P., Janssen, A.J., Smeets, R.J., Buskens, C.A., DeMeirleir, L., Van Coster, R., Baethmann, M., Voit, T., Trijbels, J.M., et al. (2000). Combined enzymatic Complex I and III deficiency associated with mutations in the nuclear encoded NDUFS4 gene. Biochem Biophys Res Commun 275, 63-68.

Chance, B., and Williams, G.R. (1955). A method for the localization of sites for oxidative phosphorylation. Nature 176, 250-254.

Chazotte, B., and Hackenbrock, C.R. (1988). The multicollisional, obstructed, long-range diffusional nature of mitochondrial electron transport. J Biol Chem 263, 14359-14367.

Chen, X.J. (2004). Sal1p, a calcium-dependent carrier protein that suppresses an essential cellular function associated With the Aac2 isoform of ADP/ATP translocase in Saccharomyces cerevisiae. Genetics 167, 607-617.

Chen, Y.-C., Taylor, E.B., Dephoure, N., Heo, J.-M., Tonhato, A., Papandreou, I., Nath, N., Denko, N.C., Gygi, S.P., and Rutter, J. (2012). Identification of a protein mediating respiratory supercomplex stability. Cell Metab 15, 348-360.

D’Aurelio, M., Gajewski, C.D., Lenaz, G., and Manfredi, G. (2006). Respiratory chain supercomplexes set the threshold for respiration defects in human mtDNA mutant cybrids. Hum Mol Genet 15, 2157-2169.

Diaz, F., Fukui, H., Garcia, S., and Moraes, C.T. (2006). Cytochrome c oxidase is required for the assembly/stability of respiratory complex I in mouse fibroblasts. Mol Cell Biol 26, 4872-4881.

Dienhart, M.K., and Stuart, R.A. (2008). The yeast Aac2 protein exists in physical association with the cytochrome bc1-COX supercomplex and the TIM23 machinery. Mol Biol Cell 19, 3934-3943.

Dudkina, N.V., Eubel, H., Keegstra, W., Boekema, E.J., and Braun, H.-P. (2005). Structure of a mitochondrial supercomplex formed by respiratory-chain complexes I and III. Proc Natl Acad Sci U S A 102, 3225-3229.

Dudkina, N.V., Kudryashev, M., Stahlberg, H., and Boekema, E.J. (2011). Interaction of complexes I, III, and IV within the bovine respirasome by single particle cryoelectron tomography. Proc Natl Acad Sci U S A 108, 15196-15200.

Dunning, C.R., McKenzie, M., Sugiana, C., Lazarou, M., Silke, J., Connelly, A., Fletcher, J.M., Kirby, D.M., Thorburn, D.R., and Ryan,
M.T. (2007). Human CIA30 is involved in the early assembly of mitochondrial complex I and mutations in its gene cause disease. EMBO J 26, 3227-3237.

Eubel, H., Jänsch, L., and Braun, H.-P. (2003). New insights into the respiratory chain of plant mitochondria. Supercomplexes and a unique composition of Complex II. Plant Physiol 133, 274-286.

Fernandez-Vizarra, E., Bugiani, M., Goffrini, P., Carrara, F., Farina, L., Procopio, E., Donati, A., Uziel, G., Ferrero, I., and Zeviani, M. (2007). Impaired Complex III assembly associated with BCS1L gene mutations in isolated mitochondrial encephalopathy. Hum Mol Genet 16, 1241-1252.

Fleischer, S., Rouser, G., Fleischer, B., Casu, A., and Kritchevsky, G. (1967). Lipid composition of mitochondria from bovine heart, liver, and kidney. J Lipid Res 8, 170-180.

Fry, M., and Green, D.E. (1981). Cardiolipin requirement for electron transfer in Complex I and III of the mitochondrial respiratory chain. J Biol Chem 256, 1874-1880.

Gawaz, M., Douglas, M.G., and Klingenberg, M. (1990). Structurefunction studies of adenine nucleotide transport in mitochondria. II. Biochemical analysis of distinct AAC1 and AAC2 proteins in yeast. J Biol Chem 265, 14202-14208.

Genova, M.L., Baracca, A., Biondi, A., Casalena, G., Faccioli, M., Falasca, A.I., Formiggini, G., Sgarbi, G., Solaini, G., and Lenaz, G. (2008). Is supercomplex organization of the respiratory chain required for optimal electron transfer activity? Biochim Biophys Acta 1777, 740-746.

Ghezzi, D., Arzuffi, P., Zordan, M., Da Re, C., Lamperti, C., Benna, C., D’Adamo, P., Diodato, D., Costa, R., Mariotti, C., et al. (2011). Mutations in TTC19 cause mitochondrial Complex III deficiency and neurological impairment in humans and flies. Nat Genet 43, 259-263.

Gohil, V.M., Hayes, P., Matsuyama, S., Schägger, H., Schlame, M., and Greenberg, M.L. (2004). Cardiolipin biosynthesis and mitochondrial respiratory chain function are interdependent. J Biol Chem 279, 42612-42618.

Gómez, L.A., Monette, J.S., Chavez, J.D., Maier, C.S., and Hagen, T.M. (2009). Supercomplexes of the mitochondrial electron transport chain decline in the aging rat heart. Arch Biochem Biophys 490, 30-35.

Gupte, S.S., and Hackenbrock, C.R. (1988). The role of cytochrome c diffusion in mitochondrial electron transport. J Biol Chem 263, 5248-5253.

Hackenbrock, C.R., Chazotte, B., and Gupte, S.S. (1986). The random collision model and a critical assessment of diffusion and collision in mitochondrial electron transport. J Bioenerg Biomembr 18, 331-368.

Hatefi, Y. (1978). Reconstitution of the electron-transport system of bovine heart mitochondria. Meth Enzymol 53, 48-54.

Hatefi, Y., Haavik, A.G., and Griffiths, D.E. (1961). Reconstitution of the electron transport system: I. Preparation and properties of the interacting enzyme complexes. Biochem Biophys Res Commun 4, 441-446.

Hatefi, Y., Haavik, A.G., and Griffiths, D.E. (1962). Studies on the electron transfer system. XL. Preparation and properties of mitochondrial DPNH-coenzyme Q reductase. J Biol Chem 237, 1676-1680.

Heinemeyer, J., Braun, H.-P., Boekema, E.J., and Kouril, R. (2007). A structural model of the cytochrome $\mathrm{C}$ reductase/oxidase supercom- 
plex from yeast mitochondria. J Biol Chem 282, 12240-12248.

Hess, D.C., Myers, C.L., Huttenhower, C., Hibbs, M.A., Hayes, A.P., Paw, J., Clore, J.J., Mendoza, R.M., Luis, B.S., Nislow, C., et al. (2009). Computationally driven, quantitative experiments discover genes required for mitochondrial biogenesis. PLoS Genet 5, e1000407.

Iwasaki, T., Matsuura, K., and Oshima, T. (1995). Resolution of the aerobic respiratory system of the thermoacidophilic archaeon, Sulfolobus sp. strain 7: I. The archaeal terminal oxidase supercomplex is a functional fusion of respiratory complexes III and IV with no ctype cytochromes. J Biol Chem 270, 30881-30892.

Jiang, F., Ryan, M.T., Schlame, M., Zhao, M., Gu, Z., Klingenberg, M., Pfanner, N., and Greenberg, M.L. (2000). Absence of cardiolipin in the crd1 null mutant results in decreased mitochondrial membrane potential and reduced mitochondrial function. J Biol Chem 275, 22387-22394.

Klingenberg, M. (1989). Molecular aspects of the adenine nucleotide carrier from mitochondria. Arch Biochem Biophys 270, 1-14.

Lazarou, M., Smith, S.M., Thorburn, D.R., Ryan, M.T., and McKenzie, M. (2009). Assembly of nuclear DNA-encoded subunits into mitochondrial Complex IV, and their preferential integration into supercomplex forms in patient mitochondria. FEBS J 276, 6701-6713.

Li, Y., D’Aurelio, M., Deng, J.-H., Park, J.-S., Manfredi, G., Hu, P., Lu, J., and Bai, Y. (2007). An assembled Complex IV maintains the stability and activity of Complex I in mammalian mitochondria. J Biol Chem 282, 17557-17562.

Marques, I., Dencher, N.A., Videira, A., and Krause, F. (2007). Supramolecular organization of the respiratory chain in Neurospora crassa mitochondria. Eukary Cell 6, 2391-2405.

McKenzie, M., Lazarou, M., Thorburn, D.R., and Ryan, M.T. (2006). Mitochondrial respiratory chain supercomplexes are destabilized in Barth Syndrome patients. J Mol Biol 361, 462-469.

Merkwirth, C., Martinelli, P., Korwitz, A., Morbin, M., Brönneke, H.S., Jordan, S.D., Rugarli, E.I., and Langer, T. (2012). Loss of prohibitin membrane scaffolds impairs mitochondrial architecture and leads to tau hyperphosphorylation and neurodegeneration. PLoS Genet 8, e1003021.

Mitchell, P., and Moyle, J. (1968). Proton translocation coupled to ATP hydrolysis in rat liver mitochondria. Euro J Biochem 4, 530-539.

Morán, M., Marín-Buera, L., Gil-Borlado, M.C., Rivera, H., Blázquez, A., Seneca, S., Vázquez-López, M., Arenas, J., Martín, M.A., and Ugalde, C. (2010). Cellular pathophysiological consequences of BCS1L mutations in mitochondrial Complex III enzyme deficiency. Hum Mutat 31, 930-941.

Moreno-Lastres, D., Fontanesi, F., García-Consuegra, I., Martín, M.A., Arenas, J., Barrientos, A., and Ugalde, C. (2012). Mitochondrial complex I plays an essential role in human respirasome assembly. Cell Metab 15, 324-335.

Muster, B., Kohl, W., Wittig, I., Strecker, V., Joos, F., Haase, W., Bereiter-Hahn, J., and Busch, K. (2010). Respiratory chain complexes in dynamic mitochondria display a patchy distribution in life cells. PLoS ONE 5, e11910.

Ogilvie, I. (2005). A molecular chaperone for mitochondrial Complex I assembly is mutated in a progressive encephalopathy. $\mathrm{J}$ Clin Invest 115, 2784-2792.

Orstavik, K.H., Orstavik, R.E., Naumova, A.K., D'Adamo, P., Gedeon, A., Bolhuis, P.A., Barth, P.G., and Toniolo, D. (1998). X chromo- some inactivation in carriers of Barth syndrome. Am J Hum Genet 63, 1457-1463.

Pagliarini, D.J., Calvo, S.E., Chang, B., Sheth, S.A., Vafai, S.B., Ong, S.-E., Walford, G.A., Sugiana, C., Boneh, A., Chen, W.K., et al. (2008). A mitochondrial protein compendium elucidates complex I disease biology. Cell 134, 112-123.

Ramírez-Aguilar, S.J., Keuthe, M., Rocha, M., Fedyaev, V.V., Kramp, K., Gupta, K.J., Rasmusson, A.G., Schulze, W.X., and Van Dongen, J.T. (2011). The composition of plant mitochondrial supercomplexes changes with oxygen availability. J Biol Chem 286, 43045-43053.

Rosca, M.G., Vazquez, E.J., Kerner, J., Parland, W., Chandler, M.P., Stanley, W., Sabbah, H.N., and Hoppel, C.L. (2008). Cardiac mitochondria in heart failure: decrease in respirasomes and oxidative phosphorylation. Cardiovasc Res 80, 30-39.

Saada, A., Edvardson, S., Rapoport, M., Shaag, A., Amry, K., Miller, C., Lorberboum-Galski, H., and Elpeleg, O. (2008). C6ORF66 is an assembly factor of mitochondrial complex I. Am J Hum Genet 82, 32-38.

Santiago, E., López-Moratalla, N., and Segovia, J.F. (1973). Correlation between losses of mitochondrial ATPase activity and cardiolipin degradation. Biochem Biophys Res Commun 53, 439-445.

Schäfer, E., Seelert, H., Reifschneider, N.H., Krause, F., Dencher, N.A., and Vonck, J. (2006). Architecture of active mammalian respiratory chain supercomplexes. J Biol Chem 281, 15370-15375.

Schägger, H., and Pfeiffer, K. (2000). Supercomplexes in the respiratory chains of yeast and mammalian mitochondria. EMBO J 19, 1777-1783.

Schägger, H., and Pfeiffer, K. (2001). The ratio of oxidative phosphorylation complexes I-V in bovine heart mitochondria and the composition of respiratory chain supercomplexes. J Biol Chem 276, 37861-37867.

Schleicher, M., Shepherd, B.R., Suarez, Y., Fernandez-Hernando, C., Yu, J., Pan, Y., Acevedo, L.M., Shadel, G.S., and Sessa, W.C. (2008). Prohibitin-1 maintains the angiogenic capacity of endothelial cells by regulating mitochondrial function and senescence. $\mathrm{J}$ Cell Biol 180, 101-112.

Sone, N., Sekimachi, M., and Kutoh, E. (1987). Identification and properties of a quinol oxidase super-complex composed of a bc1 complex and cytochrome oxidase in the thermophilic bacterium PS3. J Biol Chem 262, 15386-15391.

Strogolova, V., Furness, A., Robb-McGrath, M., Garlich, J., and Stuart, R.A. (2012). Rcf1 and Rcf2, members of the hypoxia-induced gene 1 protein family, are critical components of the mitochondrial cytochrome bc1-cytochrome c oxidase supercomplex. Mol Cell Biol 32, 1363-1373.

Stroh, A., Anderka, O., Pfeiffer, K., Yagi, T., Finel, M., Ludwig, B., and Schägger, H. (2004). Assembly of respiratory complexes I, III, and IV into NADH oxidase supercomplex stabilizes Complex I in Paracoccus denitrificans. J Biol Chem 279, 5000-5007.

Strub, G.M., Paillard, M., Liang, J., Gomez, L., Allegood, J.C., Hait, N.C., Maceyka, M., Price, M.M., Chen, Q., Simpson, D.C., et al. (2011). Sphingosine-1-phosphate produced by sphingosine kinase 2 in mitochondria interacts with prohibitin 2 to regulate Complex IV assembly and respiration. FASEB J 25, 600-612.

Sugiana, C., Pagliarini, D.J., McKenzie, M., Kirby, D.M., Salemi, R., Abu-Amero, K.K., Dahl, H.-H.M., Hutchison, W.M., Vascotto, K.A., Smith, S.M., et al. (2008). Mutation of C20orf7 disrupts complex I assembly and causes lethal neonatal mitochondrial disease. Am J 
Hum Genet 83, 468-478.

Trouillard, M., Meunier, B., and Rappaport, F. (2011). Questioning the functional relevance of mitochondrial supercomplexes by timeresolved analysis of the respiratory chain. Proc Natl Acad Sci U S A 108, E1027-1034.

Vogel, R.O., Janssen, R.J.R.J., Van den Brand, M.A.M., Dieteren, C.E.J., Verkaart, S., Koopman, W.J.H., Willems, P.H.G.M., Pluk, W., Van den Heuvel, L.P.W.J., Smeitink, J.A.M., et al. (2007). Cytosolic signaling protein Ecsit also localizes to mitochondria where it interacts with chaperone NDUFAF1 and functions in Complex I assembly. Genes Dev 21, 615-624.

Vreken, P., Valianpour, F., Nijtmans, L.G., Grivell, L.A., Plecko, B., Wanders, R.J.A., and Barth, P.G. (2000). Defective remodeling of cardiolipin and phosphatidylglycerol in barth syndrome. Biochem Biophy Res Commun 279, 378-382.

Wang, J., Cao, Y., Chen, Y., Chen, Y., Gardner, P., and Steiner, D.F. (2006). Pancreatic $\beta$ cells lack a low glucose and O2-inducible mitochondrial protein that augments cell survival. Proc Natl Acad Sci U S A 103, 10636-10641.

Wenz, T., Hielscher, R., Hellwig, P., Schägger, H., Richers, S., and Hunte, C. (2009). Role of phospholipids in respiratory cytochrome bc(1) complex catalysis and supercomplex formation. Biochim Biophys Acta 1787, 609-616.

Zhang, M., Mileykovskaya, E., and Dowhan, W. (2005). Cardiolipin is essential for organization of complexes III and IV into a supercomplex in intact yeast mitochondria. J Biol Chem 280, 29403-29408. 